\title{
Control of Aggregate Size of Polyethyleneimine-Coated Magnetic Nanoparticles for Magnetofection
}

\author{
Xiaoliang Wang, Linzhu Zhou, Yongjie Ma, Xu Li, and Hongchen Gu（ $\square ）$ \\ Nano Biomedicine Research Center, Med-X Research Institute, Shanghai Jiaotong University, 1954 Huashan Road, Shanghai \\ 200030, China
}

Received: 23 November 2008 / Revised: 25 February 2009 / Accepted: 1 March 2009

(C)Tsinghua University Press and Springer-Verlag 2009. This article is published with open access at Springerlink.com

\begin{abstract}
Using magnetic nanoparticles to enhance gene transfection, a recently developed technique termed magnetofection, has been shown to be a powerful technology in gene delivery. The most widely used magnetic nanoparticles in this area are those coated with polyethyleneimine, which is a well known nonviral transfection agent. In this article, we report methods to control the aggregate size of polyethyleneiminecoated magnetite particles. These particles were then used to enhance transfection of green fluorescent protein (GFP) into NIH 3T3 cells in vitro. We find that the aggregate size of the particles has a great effect on their performance in magnetofection, with less aggregated magnetic particles being more effective in enhancing the gene transfection.
\end{abstract}

\section{KEYWORDS}

Magnetofection; transfection; magnetic nanoparticles, polyethyleneimine (PEI)

\section{Introduction}

Many viral and non-viral gene vectors have been developed to deliver nucleic acids into cells, which is one of the main challenges in gene therapy [1-3]. However, safe, reliable, and effective gene delivery is yet to be achieved, especially in vivo [4]. Efficiency of the delivery is largely determined by the process by which genes move to, and contact with, the target cells, which is a diffusion-limited process under standard cell culture conditions [5]. To speed up the process, a new technology termed "magnetofection" has been developed [6-8]. In this method, the complexes of nucleic acids and their vectors are combined with magnetic nanoparticles, so they can be drawn and concentrated to the target cells by applying a magnetic field. Via magnetofection, the efficiency of certain gene vectors can be enhanced by up to several-hundred-fold. Moreover, the process time can be reduced from the $4 \mathrm{~h}$ required for standard procedures to $15 \mathrm{~min}$.

In magnetofection, the magnetic nanoparticles require suitable surface functionalization to couple with the gene complexes. Leary et al. have used streptavidin-coated magnetic nanoparticles to tether biotin-labeled polymerase chain reaction (PCR) products [9]. The resulting complexes have much greater efficiency than ones labeled only with

Address correspondence to hcgu@sjtu.edu.cn 
biotin. Besides bioconjugation, a much more simple method involves using positively charged magnetic particles to couple with nuclear acids via electrostatic interactions [10,11]. One of the most widely used positively charged modification agents is polyethyleneimine (PEI) which is capable of forming complexes with DNA and condensing them into compact nanoparticles $[12,13]$. Moreover, PEI can destabilize the endosomal membrane by the so-called "proton sponge effect" to enhance DNA release from endosomes and protect DNA against degradation [14]. To date, PEI is one of the most efficient nonviral transfection agents.

Several groups have reported various methods of preparation of PEI-coated magnetic particles (MP-PEI) for gene transfections. For example, Plank and co-workers synthesized magnetic particles in situ in PEI solutions [8, 15, 16], Hottiger and coworkers directly mixed maghemite dispersions with PEI solutions [10], whilst McBain and co-workers exploited covalent bonds to attach PEI to magnetic particles [17]. The MP-PEI prepared by these methods are all capable of enhancing gene delivery.

All the above MP-PEI are actually used in the form of aggregates, ranging from tens to hundreds of nanometers. We know that the size of transfection agents dramatically influences their performance because the endocytosis rate, cytoxicity, and the velocity of cytoplasmatic movement are all functions of their size $[18,19]$. In magnetofection, the size of the magnetic particle aggregates also influences the magnetic force of attraction which is one of the most important factors contributing to enhancement of gene transfections. However, there have been no reports of how to control the size of magnetic particle aggregates and its effect on the efficiency of magnetofection.

In the present study, PEI is used both as a gene vector and the modification agent for the magnetic particles. We prepared MP-PEI via electrostatic adsorption of PEI to negatively charged magnetic particles. The aggregate size of as-prepared MP-PEI was adjusted by changing the ratio of PEI to magnetic particles. The effect of varying $\mathrm{pH}$ on aggregate size was also investigated. The ability of MP-PEI with various diameters to enhance transfection of porcine epidermal growth factor (pEGF) DNA to NIH-3T3 (mouse embryonic fibroblast cell line) was studied in vitro.

\section{Materials and methods}

\subsection{Materials}

PEI 25k was purchased from Aldrich. Lipofectamine $^{\text {TM }} 2000$ Transfection Reagent, RPMI (Roswell Park Memorial Institute) tissue culture medium and fetal calf serum were purchased from Invitrogen. The pEGFP-C1 plasmid (Clontech) was amplified in E. coli (strain DH5 $\alpha$ ) and purified using an endotoxin-free Maxi-prep plasmid kit (Qiagen). All other reagents were obtained from Shanghai Reagent Company.

\subsection{Preparation of MP-PEI}

Magnetite nanoparticles (MP) were synthesized by co-precipitation of ferric and ferrous salts in a basic solution. For pre-modification by citric acid, asprepared magnetite nanoparticles $(0.69 \mathrm{~g})$ and citric acid (CA) monohydrate $(0.2 \mathrm{~g})$ were both added into $20 \mathrm{~mL}$ of deionized water. The mixture was sonicated for $40 \mathrm{~min}$, and then neutralized by 0.5 $\mathrm{mol} / \mathrm{L} \mathrm{NaOH}$ solution. In order to remove excess electrolytes, the neutralized dispersion was subjected to dialysis against deionized water in a $12-14 \mathrm{kD}$ cutoff cellulose tube for $48 \mathrm{~h}$. Finally, the dispersion of CA stabilized particles (MP-CA) was centrifuged at $9500 \mathrm{rpm}$ for $20 \mathrm{~min}$. The pellet was discarded and the dispersion was used for subsequent operations.

For PEI coating, the above dispersion was mixed with different amounts of $5 \%(\mathrm{~m} / \mathrm{m})$ PEI solution. After being gently shaken for $3 \mathrm{~min}$, the mixture was then neutralized with $0.5 \mathrm{~mol} / \mathrm{L} \mathrm{HCl}$ solution. Asprepared PEI-coated magnetic particles (MP-PEI-N) were washed three times by magnetic separation in order to remove unbound PEI and salts.

\subsection{Acidification and subsequent neutralization of MP-PEI}

The dispersion of MP-PEI-N was acidified to $\mathrm{pH}=2$ by $0.5 \mathrm{~mol} / \mathrm{L} \mathrm{HCl}$ solution and kept for $10 \mathrm{~min}$ before being neutralized by $0.5 \mathrm{~mol} / \mathrm{L} \mathrm{NaOH}$ solution. 
The PEI-coated magnetic particles after this process (MP-PEI-A) were washed three times by magnetic separation.

\subsection{Characterization}

The zeta potentials of the magnetic nanoparticles as a function of dispersion $\mathrm{pH}$ were measured by a Zetasizer 2000 (Malvern, UK). The amount of PEI on the particle surface was measured by thermogravimetric analyses (TGA) with a heating rate of $10{ }^{\circ} \mathrm{C} / \mathrm{min}$ in a flowing argon atmosphere using a TGA2050 instrument (TA, USA). The morphology and size of the samples were examined by transmission electron microscopy (TEM) using a JEM-2100 microscope (JEOL, Japan). The aggregate sizes of PEI modified particles in dispersions were directly investigated via dynamic light scattering (DLS) using an HPPS instrument (Malvern, UK).

\subsection{GFP reporter expression in NIH-3T3 cells}

NIH-3T3 cells were cultured in RPMI medium supplemented with $10 \%$ fetal calf serum, $100 \mathrm{U} / \mathrm{cm}^{3}$ penicillin, $0.1 \mathrm{mg} / \mathrm{cm}^{3}$ streptomycin, $0.25 \mathrm{mg} / \mathrm{cm}^{3}$ amphotericin B, and $2 \mathrm{mmol} / \mathrm{L}$ L-glutamine.

For transfection studies, cells were seeded at $2 \times 10^{4}$ cells per well in 6-well tissue culture plates and incubated overnight at $37{ }^{\circ} \mathrm{C}$ with $5 \% \mathrm{CO}_{2}$. The medium was removed from the cells and replaced with $1 \mathrm{~mL}$ of serum-free culture medium containing $3 \mu \mathrm{g}$ of GFP, $2.4 \mu \mathrm{g}$ of MPPEI, and the desired amount of 0.26 $\mathrm{mg} / \mathrm{mL}$ PEI solution ( $1 \mu \mathrm{g}$ of DNA corresponds to $3 \mathrm{nmol}$ of phosphate, and $1 \mu \mathrm{L}$ of PEI solution corresponds to $6 \mathrm{nmol}$ of amine nitrogen) [12]. The cell culture plates were then placed on a Nd-Fe-B permanent magnet for $15 \mathrm{~min}$. The medium was removed from each well and replaced with 0.5 $\mathrm{cm}^{3}$ of RPMI medium supplemented with $10 \%$ fetal calf serum, $100 \mathrm{U} / \mathrm{cm}^{3}$ penicillin, $0.1 \mathrm{mg} / \mathrm{cm}^{3}$ streptomycin, $0.25 \mathrm{mg} / \mathrm{cm}^{3}$ amphotericin $\mathrm{B}$, and 2 $\mathrm{mmol} / \mathrm{L}$ L-glutamine. Lipofectamine
$2000^{\mathrm{TM}}$ transfection was performed using $3 \mu \mathrm{g}$ DNA per well following the manufacturer's recommended protocols. Cells were subsequently harvested $24 \mathrm{~h}$ post-transfection and analyzed for GFP expression by flow cytometry (FACSCalibur; BD Biosciences), with 10,000 cells being tested every time. All experiments were performed in triplicate. The cells subjected to magnetofection or normal PEI transfection were also investigated by fluorescence microscopy (IX71, Olympus).

\section{Results and discussion}

Characterization data for MP-CA are shown in Fig. 1. TEM showed that the magnetic particles prepared by co-precipitation had an average diameter around $10 \mathrm{~nm}$ (Fig. 1(a)). After modification with citric acid, the magnetic particles were strongly negatively charged. Their zeta potentials reached as low as -45 $\mathrm{mV}$ in neutral conditions (Fig. 1(b)). Citric acid has three carboxyl groups which facilitates its adsorption onto the iron oxide particles [18], whilst ionization of the carboxyl groups provides the coated magnetic particles with a negative charge. Therefore, the

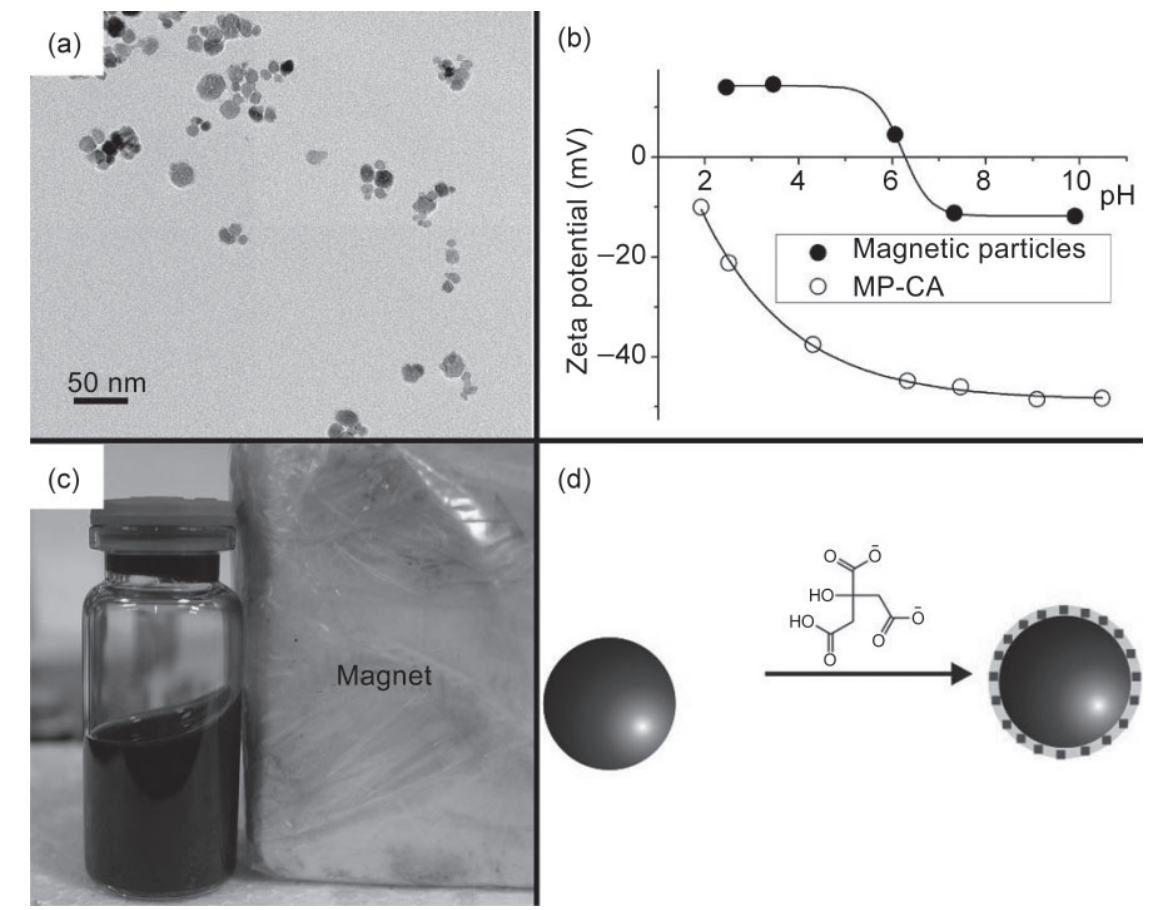

Figure 1 (a) TEM image of MP-CA; (b) zeta potentials of magnetic particles and MP-CA as a function of dispersion $\mathrm{pH}$ (which was adjusted by $0.1 \mathrm{~mol} / \mathrm{L} \mathrm{HCl}$ or $\mathrm{NaOH}$ ); (c) photo of MPCA dispersion; (d) schematic representation of the CA coating 
particles give a stable dispersion in water due to strong mutual electrostatic repulsion (Fig. 1(c)). Moreover, these negatively charged particles (Fig. 1(d)) are able to adsorb cationic polyelectrolytes such as PEI.

After mixing with the PEI solutions, the surface charge of the magnetic particles changed from highly negative to highly positive, and at the same time, aggregates of MP-PEI formed (Fig. 2). Their positive zeta potential and aggregate size are both functions of the weight ratio of PEI to $\mathrm{Fe}_{3} \mathrm{O}_{4}$. Higher ratios lead to more positive zeta potentials and smaller aggregates of MP-PEI, which is consistent with the results reported by Petri-Fink and co-workers [11]. By changing the weight ratio, we could control the zeta potential in the range +10 to $+50 \mathrm{mV}$, and the aggregate size in the range 200 to $800 \mathrm{~nm}$.

The de-aggregation of MP-PEI-N in acid conditions was studied by DLS as shown in Fig. 3(a). The aggregate size of MP-PEI starts to decrease at $\mathrm{pH}=4$ and reaches ca. $80 \mathrm{~nm}$ at $\mathrm{pH}=2$. Interestingly, neutralization of the dispersions did not lead to reaggregation of the magnetic particles; therefore, the de-aggregation in acid condition was an irreversible process. This process was also demonstrated by the peak shifts in the aggregate size distribution curves, as shown in the Electronic Supplementary Material

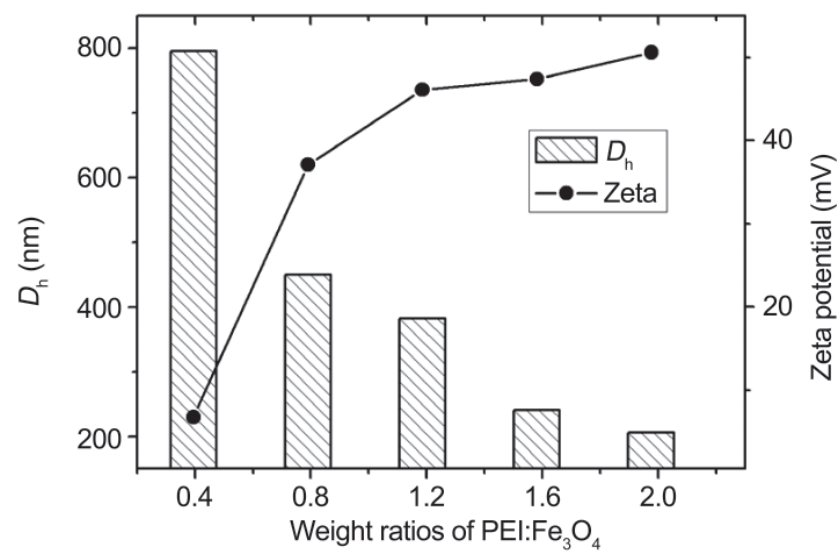

Figure 2 Hydrodynamic diameters $\left(D_{h}\right)$ and zeta potentials of MPPEI-N as a function of weight ratio of PEI to magnetite. The particles were all pre-neutralized to $\mathrm{pH}=7$ with $0.1 \mathrm{~mol} / \mathrm{L} \mathrm{HCl}$ solution

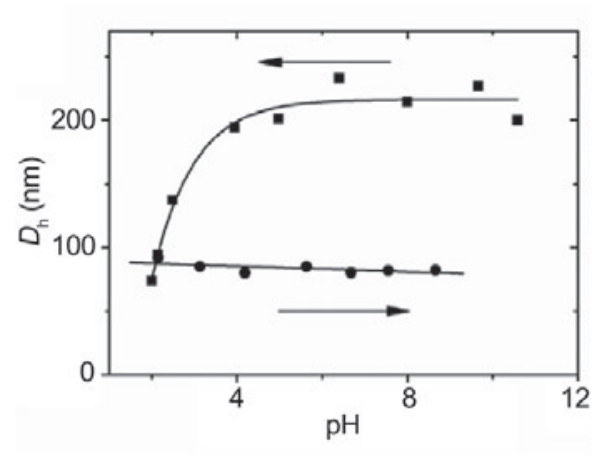

(a)

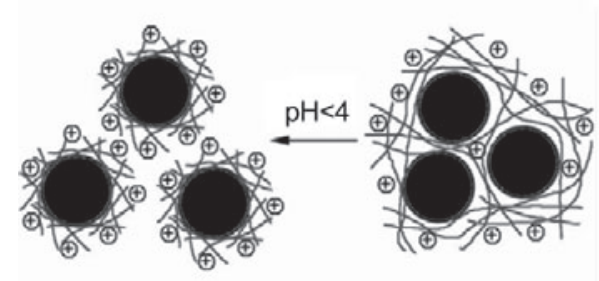

(c)

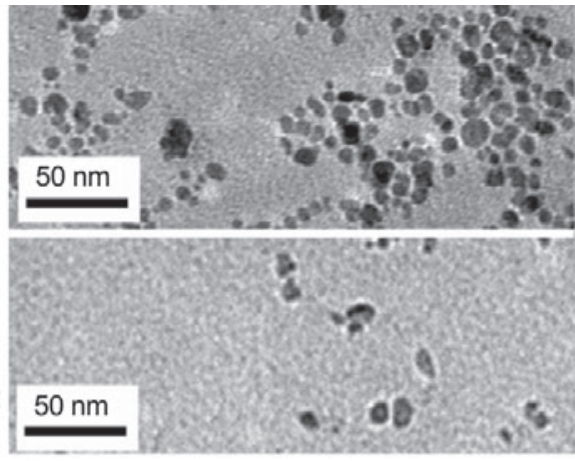

(b)

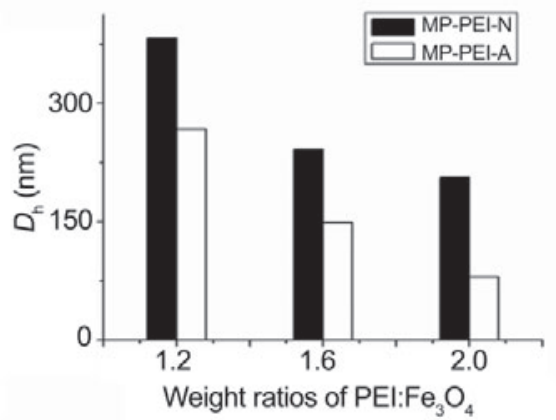

(d)

Figure 3 (a) $D_{\mathrm{h}}$ of MP-PEI (weight ratio of PEI to magnetite $=2$ ) at varying $\mathrm{pH}$ adjusted from the original basic to acid, and then back to basic; (b) TEM images of the MP-PEI-N (upper) and MP-PEI-A (lower) when the weight ratio was 2.0; (c) schematic representation of MP-PEI de-aggregation in acid conditions; (d) $D_{\mathrm{h}}$ of MP-PEI-N and MP-PEI-A as a function of weight ratio of PEl to magnetic particles 
(ESM, Fig. S-1). PEI contains a large number of amino groups and becomes increasingly protonated with decreasing $\mathrm{pH}$. The electrostatic repulsion between MP-PEI aggregates was therefore enhanced at low $\mathrm{pH}$, leading to de-aggregation. The process is also confirmed by the TEM images (Fig. 3(b) and Fig.S-2 in the ESM). In acid conditions, the molecular chains of PEI also became more stretched and increased the steric repulsion between particles. Therefore, after acidification there were both electrostatic and steric interactions between the particles (Fig.3(c)). Thus, re-neutralization of the MP-PEI did not result in reaggregation. Based on this finding, we can prepare neutral dispersions of weakly-aggregated MP-PEI (Fig. 3(d)) for subsequent applications.

To determine the amount of modification agent on the particle surface, TGA curves of MP-CA, MPPEI-N, and MP-PEI-A were obtained. As shown in Fig. 4, MP-PEI-N has a larger weight loss than MPCA $(17 \%$ vs $6 \%)$, which confirms that PEI was coated on the surface. The MP-PEI-A and MP-PEI-N have almost the same amount of PEI, which means the acidification process at $\mathrm{pH}=2$ for $10 \mathrm{~min}$ did not lead to leaching of PEI from the magnetic nanoparticles. Consequently, the changes in diameter of MP-PEI could not be due to loss of the coating polymer from their surface. The results confirm that de-aggregation of MP-PEI occurred in acid conditions.

Because the magnetic nanoparticle diameters decreased irreversibly after acidification, MP-PEI-N and MP-PEI-A also form complexes of different size with DNA and PEI, as shown in Fig. 5. In general, the diameter of the MP-PEI-N/DNA/PEI complex is larger than that of the corresponding MP-PEI-A. We know that the size of a DNA complex dramatically affects its transfection efficiency. In cases such as lipoplexes and polyplexes, smaller complexes have better performances than larger ones [21, 22]. Therefore, decreasing the diameter of MP-PEI aggregates should improve their magnetofection efficiency.

The magnetofection efficiencies of PEI 25k enhanced by MP-PEI-N and MP-PEI-A were evaluated in NIH-3T3 cells, with standard Lipofectamine $2000^{\mathrm{TM}}$ and PEI transfection as two control experiments. The results are shown in Fig.

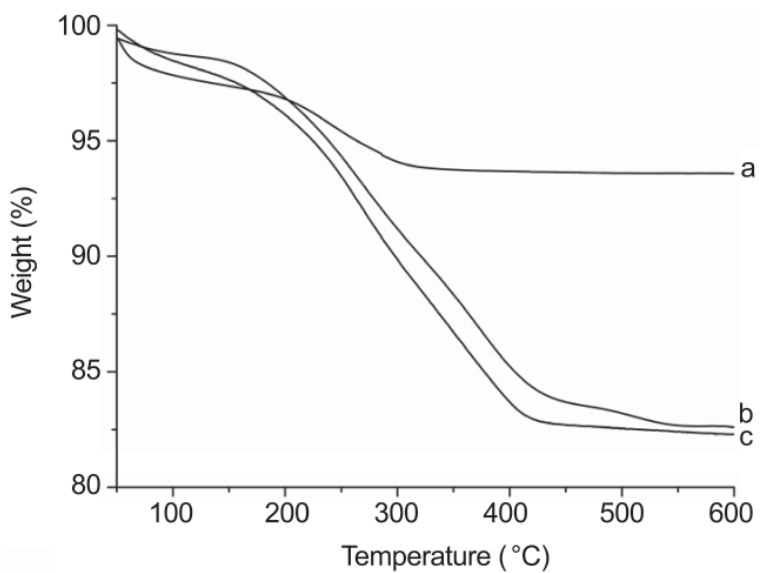

Figure 4 TGA curves of (a) MP-CA, (b) MP-PEI-A when the weight ratio of PEI to magnetite was 2 and (c) MP-PEI-N when the weight ratio was 2

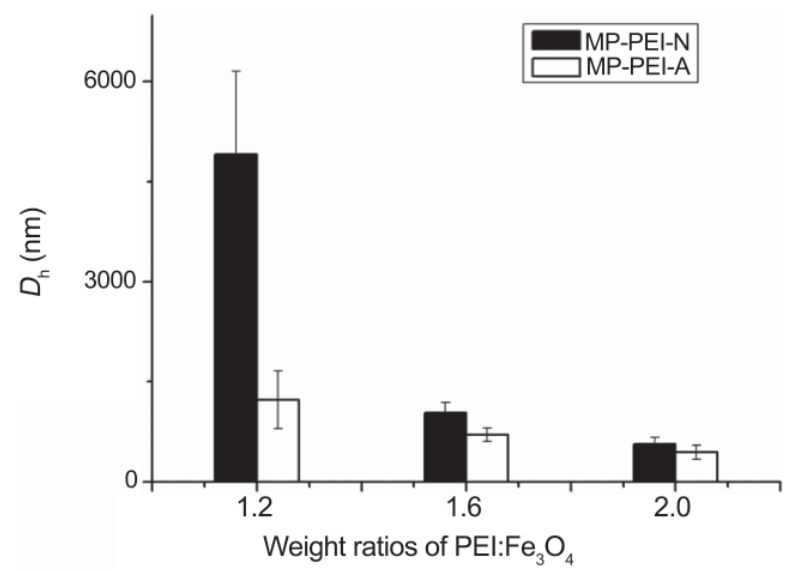

Figure $5 D_{\mathrm{h}}$ of MP-PEI/DNA/PEl complexes using MP-PEl prepared with weight ratios of $\mathrm{PEI}$ to magnetite of $1.2,1.6$ and 2.0

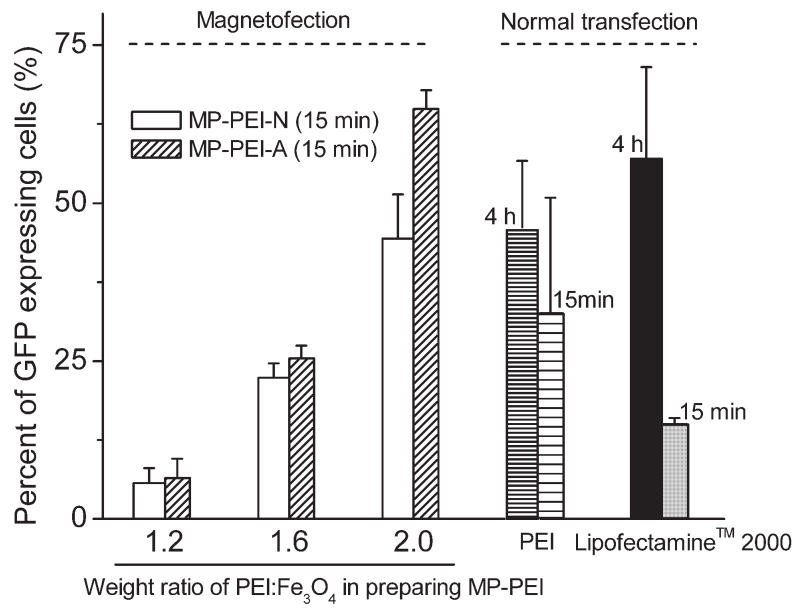

Figure 6 Transfection efficiencies of GFP plasmid to NIH 3T3 cells with Lipofectamine $2000^{\mathrm{TM}}$ for $4 \mathrm{~h}$, PEl for $4 \mathrm{~h}$ and magnetofection for 15 min using MP-PEl prepared with weight ratio of PEI to magnetite of $1.2,1.6$, and 2.0 . The molar ratio of $\mathrm{PEI}$ nitrogen to DNA phosphate was 20 in all the experiments with PEI 
6. The transfection efficiency of MP-PEI particles increased with increasing weight ratio of PEI to magnetite. For a given ratio, MP-PEI-A had a higher transfection efficiency than that of MP-PEI-N.

The particles were all prepared from the same batch of MP-CA; therefore, their differences in colloidal properties only result from the different weight ratios of PEI to magnetite used in the PEI coating process. MP-PEI with ratios of 1.2, 1.6, and 2.0 have almost the same zeta potentials (Fig. 2). In addition, the de-aggregation process did not change the amount of PEI on the particle surface. Therefore, it is certain that the differences in transfection efficiency result from the difference in aggregate size of the magnetic particles. Generally speaking, MP-PEI with smaller aggregate size had better magnetofection performance than that of larger particles. Using MP-PEI-A prepared with a weight ratio of 2.0, magnetofection for $15 \mathrm{~min}$ showed a higher percentage of GFP expression cells that obtained by standard PEI transfection for $4 \mathrm{~h}$, while the efficiencies were quite low for the severely aggregated particles. Therefore, because aggregate sizes has such an important effect, not all kinds of PEI coated magnetic nanoparticles are suitable for magnetofection.

It is interesting that when MP-PEI-A was added but the cell culture plate was not placed on the magnetic plate, the efficiency was only $24.6 \%$ (datum not shown in Fig. 6), lower than the transfection with PEI alone. This is in accordance with the previous report from Kamau et al. [10]. Because the transfections of the cells were only enhanced when placed on a magnetic plate, it is clear that the key role of MP-PEI in magnetofection lies in drawing the DNA-PEI complexes onto the surface of the cells via the magnetic force.

Evidence of protein expression enhancement by weakly aggregated MP-PEI was also observed by fluorescence microscopy, which indicated that cells after magnetofection exhibited noticeably higher fluorescence compared with standard PEI transfected cells (Fig. 7). These observations demonstrate that although the percentage of transfected cells did not increase dramatically, the proteins expression levels were great improved after magnetofection. This phenomenon is probably due to the fact that flow cytometry is a very sensitive instrument; it can detect and count all the cells expressing green fluorescent protein, no matter whether the signal is strong or

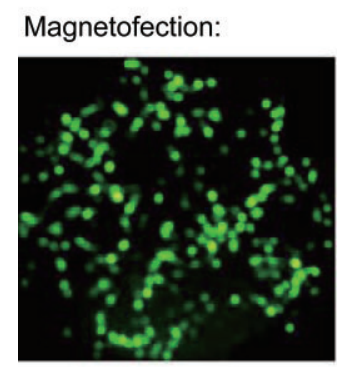

$\mathrm{N} / \mathrm{P}=15$

PEI transfection:

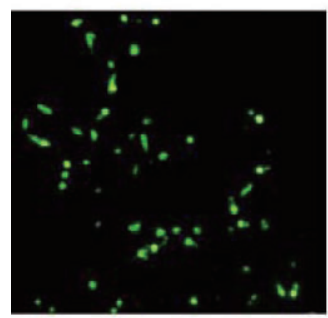

$N / P=15$

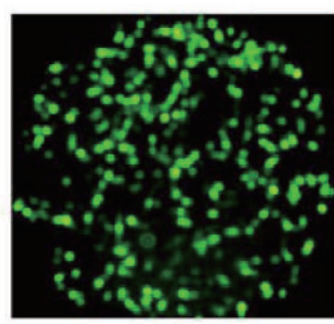

$\mathrm{N} / \mathrm{P}=\mathbf{2 0}$

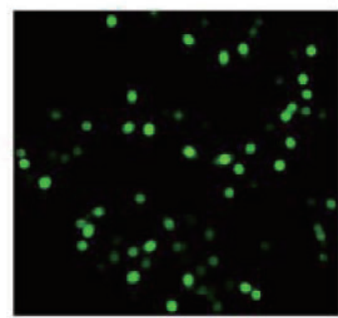

$\mathrm{N} / \mathrm{P}=20$

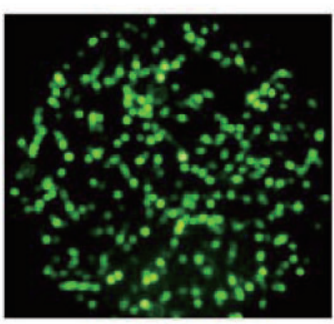

$\mathrm{N} / \mathrm{P}=25$

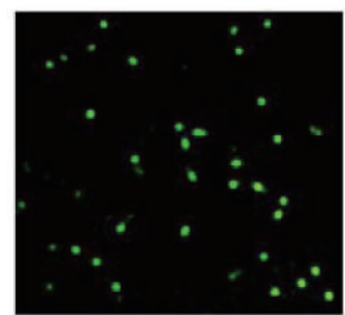

$\mathrm{N} / \mathrm{P}=25$

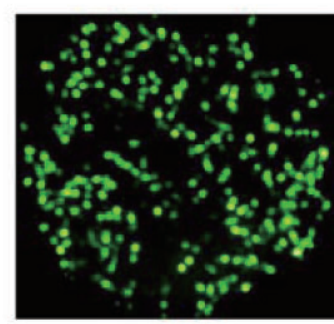

$\mathrm{N} / \mathrm{P}=\mathbf{3 0}$

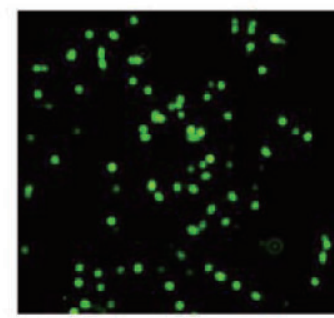

$N / P=30$

Figure 7 Expression of green fluorescent protein (GFP) in NIH 3 T3 cells transfected with GFP seen through a fluorescence microscope. The lower row shows the effect of standard PEI transfection for $4 \mathrm{~h}$; the upper row shows the effect of magnetofection for 15 min using $80 \mathrm{~nm}$ MP-PEI-A prepared with a weight ratio of PEI to magnetite of 2.0. N/P means molar ratio of PEl nitrogen to DNA phosphate in the experiments 
weak.

Although both PEI and liposome have been successfully applied numerous times to deliver gene materials into cells in vitro, their cytotoxicities limit their applications in practice. In contrast, as shown in Fig. 8, magnetofection has considerably lower cytotoxicity than the standard PEI and liposome transfections. We have mentioned above that cells needed incubation in serum-free and PEI-containing medium for $4 \mathrm{~h}$ in standard PEI transfection, while they needed only $15 \mathrm{~min}$ under such conditions in magnetofection. This difference can probably explain why the magnetofection did less harm to the cells.

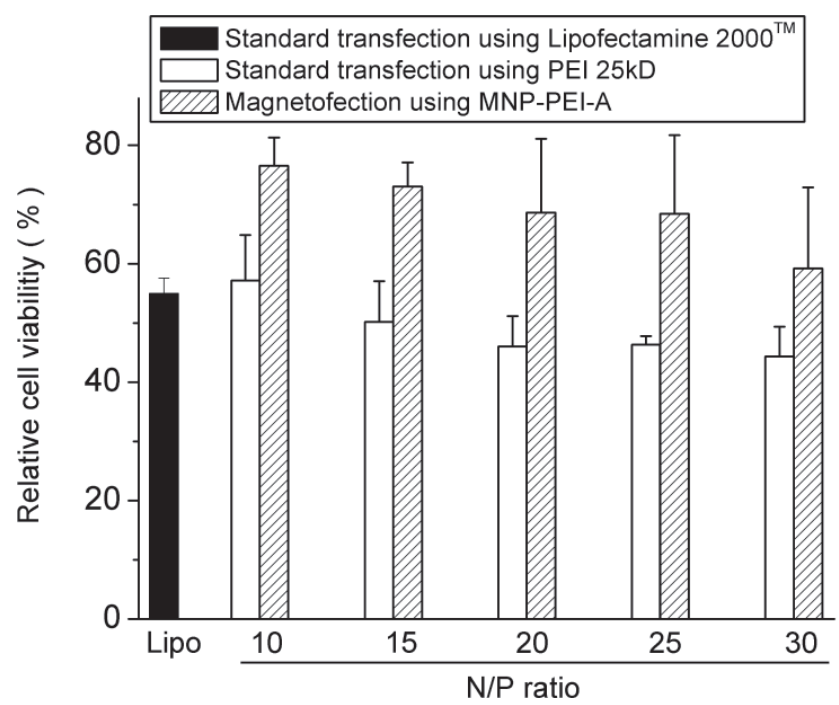

Figure 8 Effects of transfection and magnetofection on cell viabilities as detected using an MTT cytotoxicity assay kit $(n=8)$

\section{Summary}

In this paper, we have presented a simple method to prepare PEI-coated magnetic nanoparticles, the aggregate sizes of which are determined by the weight ratio of PEI to magnetic nanoparticles. The prepared particles de-aggregated in acid conditions but did not re-aggregate after neutralization. Utilizing this discovery, we prepared de-aggregated PEI-coated magnetic particles which had smaller colloidal diameters than the original particles but nearly the same surface potentials and amount of PEI. In transfection of GFP plasmid to NIH-3T3 cells, MP-PEI prepared with a higher weight ratio of PEI to magnetic nanoparticles showed better transfection performance. De-aggregation of MP-PEI after acidification led to improved transfection efficiencies. Based on all these results, we conclude that the size of magnetic nanoparticle aggregates has a dramatic effect on their performance in magnetofection. Only those weakly aggregated particles can successfully enhance gene transfection. Our conclusions provide a new criterion of aggregate size for screening magnetic nanoparticles for use in magnetofection. We also found that magnetofection was much less cytotoxic than standard transfection.

\section{Acknowledgements}

This work was supported by National 973 Program (2006CBON0300) and International Cooperation Project $(20080068,075207012)$. The authors would like to thank the Instrumental Analysis Center of Shanghai Jiaotong University for materials characterization.

Electronic Supplementary Material: Supplementary material is available in the online version of this article at http://dx.doi.org/10.1007/s12274-009-9035-6 and is accessible free of charge.

\section{References}

[1] Li, W.; Szoka, F. C. Lipid-based nanoparticles for nucleic acid delivery. Pharm. Res. 2007, 24, 438-449.

[2] Wagner, E.; Kloeckner, J. Gene delivery using polymer therapeutics. Adv. Polym. Sci. 2006, 192, 135-173.

[3] Carter, P. J.; Samulski, R. J. Adeno-associated viral vectors as gene delivery vehicles. Int. J. Mol. Med. 2000, 6, 1727.

[4] Pouton, C. W.; Seymour, L. W. Key issues in non-viral gene delivery. Adv. Drug Deliver. Rev. 2001, 46, 187 203.

[5] Luo, D.; Saltzman, W. M. Enhancement of transfection by physical concentration of DNA at the cell surface. Nat. Biotechnol. 2000, 18, 893-895.

[6] Mah, C.; Fraites, T. J.; Jr.; Zolotukhin, I.; Song, S.; Flotte, T. R.; Dobson, J.; Batich, C.; Byrne, B. J. Improved method of recombinant AAV2 delivery for systemic targeted gene therapy. Mol. Ther. 2002, 6, 106-112. 
[7] Scherer, F.; Anton, M.; Schillinger, U.; Henkel, J.; Bergemann, C.; Kruger, A.; Gansbacher, B.; Plank, C. Magnetofection: Enhancing and targeting gene delivery by magnetic force in vitro and in vivo. Gene. Ther. 2002, 9, 102-109.

[8] Mykhaylyk, O.; Antequera, Y. S.; Vlaskou, D.; Plank, C. Generation of magnetic nonviral gene transfer agents and magnetofection in vitro. Nat. Protoc. 2007, 2, 2391 $-2411$.

[9] Prow, T.; Smith, J. N.; Grebe, R.; Salazar, J. H.; Wang, N.; Kotov, N.; Lutty, G.; Leary, J. Construction, gene delivery, and expression of DNA tethered nanoparticles. Mol. Vis. 2006, 12, 606-615.

[10] Kamau, S. W.; Hassa, P. O.; Steitz, B.; Petri-Fink, A.; Hofmann, H.; Hofmann-Amtenbrink, M.; von Rechenberg, B.; Hottiger, M. O. Enhancement of the efficiency of non-viral gene delivery by application of pulsed magnetic field. Nucleic Acids Res. 2006, 34, e40.

[11] Steitz, B.; Hofmann, H.; Kamau, S. W.; Hassa, P. O.; Hottiger, M. O.; von Rechenberg, B.; HofmannAmtenbrink, M.; Petri-Fink, A. Characterization of PEIcoated superparamagnetic iron oxide nanoparticles for transfection: Size distribution, colloidal properties and DNA interaction. J. Magn. Magn. Mater. 2007, 311, 300 -305 .

[12] Boussif, O.; Lezoualc'h, F.; Zanta, M. A.; Mergny, M. D.; Scherman, D.; Demeneix, B.; Behr, J. P. A versatile vector for gene and oligonucleotide transfer into cells in culture and in vivo: Polyethylenimine. Proc. Natl. Acad. Sci. USA 1995, 92, 7297-7301.

[13] Dunlap, D. D.; Maggi, A.; Soria, M.;, Monaco, L. Nanoscopic structure of DNA condensed for gene delivery. Nucleic Acids Res. 1997, 25, 3095-3101.

[14] Remy-Kristensen, A.; Clamme, J. P. Vuilleumier, C.; Kuhry,
J. G.; Memy, Y. Role of endocytosis in the transfection of L929 fibroblasts by polyethylenimine/DNA complexes. BBA-Biomembranes 2001, 1514, 21-32.

[15] Gersting, S. W.; Schillinger, U.; Lausier, J.; Nicklaus, P.; Rudolph, C; Plank, C.; Reinhardt, D.; Rosenecker, J. Gene delivery to respiratory epithelial cells by magnetofection. J. Gene. Med. 2004, 6, 913-922.

[16] Huth, S.; Lausier, J.; Gersting, S. W.; Rudolph, C.; Plank, C.; Welsch, U.; Rosenecker, J. Insights into the mechanism of magnetofection using PEI-based magnetofectins for gene transfer. J. Gene. Med. 2004, 6, 923-936.

[17] McBain, S. C.; Yiu, H. H. P.; Haj, A. E.; Dobson, J. Polyethyleneimine functionalized iron oxide nanoparticles as agents for DNA delivery and transfection. J. Mater. Chem. 2007, 17, 2561-2565.

[18] Xu, Z. P.; Zeng, Q. H.; Lu, G. Q,; Yu, A. B. Inorganic nanoparticles as carriers for efficient cellular delivery. Chem. Eng. Sci. 2006, 61, 1027-1040.

[19] Prabha, S.; Zhou, W. Z.; Panyam, J.; Labhasetwar, V. Sizedependency of nanoparticle-mediated gene transfection: Studies with fractionated nanoparticles. Int. J. Pharm. 2002, 244, 105-115.

[20] Yu, S.; Chow, G. M. Carboxyl group $\left(-\mathrm{CO}_{2} \mathrm{H}\right)$ functionalized ferrimagnetic iron oxide nanoparticles for potential bio-applications. J. Mater. Chem. 2004, 14, 2781-2786.

[21] Ross, P. C.; Hui, S. W. Lipoplex size is a major determinant of in vitro lipofection efficiency. Gene Ther. 1999, 6, 651-659.

[22] Choosakoonkriang, S.; Lobo, B. A.; Koe, G. S.; Koe, J. G.; Middaugh, C. R. Biophysical characterization of PEl/DNA complexes. J. Pharm. Sci. 2003, 92, 1710-1722. 